2. Алибеков А.К., Гасанов К.А. Проектирование каналов в земляном русле с оптимальными параметрами: Учебное пособие. - Махачкала: ФГБОУ ВПО «ДГТУ», 2013.

3. Дементьев В.Г. Орошение. - М.: Колос, 1979. - 304 с.

4. Михалев М.А., Алибеков А. К. Условие начала трогания несвязных грунтов. В кн.: Гидравлика русловых потоков: Сб.науч.тр. - Калинин: КГУ: 1985. - C. 8 - 14.

5. Михалев М.А. Распределение скорости течения по живому сечению каналов различного поперечного сечения при равномерном движении. Сб. науч. тр. ЛПИ. Л.: ЛПИ, 1982, № 383. - С. 8 - 15.

6. Алибеков Г.А. Математическое моделирование максимальной длины гидравлических каналов в естественном русле с помощью ЭВМ// Вестник Дагестанского государственного технического университета. Технические науки. - 2014. - № 2(33). - C. 31-40.

УДК 620.98

Герейханов Р.К., Магомедов А.М.

СПОСОБ ПОВЫШЕНИЯ ТОЧНОСТИ ИЗМЕРЕНИЙ ИНТЕЛЛЕКТУАЛЬНЫХ СИСТЕМ УЧЕТА ЭЛЕКТРОЭНЕРГИИ

Gereykhanov R.K., Magomedov A.M.

\title{
METHOD FOR INCREASING THE ACCURACY OF MEASUREMENT INTELLIGENT ELECTRICITY METERING SYSTEMS
}

В данной статье рассматриваются вопросы повышения точности измерений первичных средств сбора информации, проводится анализ методов автоматической коррекиии погрешностей, на основе которых предлагается решение проблем, связанных с увеличением точности устройств сбора и учета электроэнергии.

Ключевые слова: точность измерений, электроэнергия, погрешность, алгоритм.

This article discusses the issues of improving the accuracy of measurements of the primary means of gathering information, the analysis of methods for automatic correction of errors on the basis of which the proposed solution to the problems associated with increasing the accuracy of data collection devices and electricity metering.

Key words: accuracy of measurements, electric power, error, algorithm. 
Качество электроэнергии влияет на работоспособность и эффективность функционирования питаемого электрооборудования. Показатели и нормы качества электрической энергии в электрических сетях систем электроснабжения общего назначения, находящиеся в собственности различных потребителей, устанавливаются ГОСТом 13109-97 [1].

Существуют следующие основные показатели качества электроэнергии:

1. Отклонение частоты - характеризуется разностью действительного и номинального значений переменного тока в системе электроснабжения;

2. Отклонение напряжения - показатель установившегося отклонения текущего значения напряжения от номинального;

3. Колебания напряжения - характеризуются размахом изменения напряжения, частотой повторения изменений напряжения, интервалом между изменениями напряжения и дозой фликера - мерой восприимчивости человека к воздействию колебаний светового потока искусственных источников освещения, вызванных колебаниями напряжения в электрической сети за установленный промежуток времени;

4. Несинусоидальность напряжения, обусловленная появлением в кривой напряжения помимо гармоники основной частоты гармоники других высших частот, кратных основной частоте. Возникновение несинусоидальности напряжения объясняется наличием потребителей электроэнергии с нелинейной вольт-амперной характеристикой (в основном тиристорные преобразователи электрической энергии);

5. Провал напряжения - характеризуется глубиной и длительностью. Это внезапное существенное снижение напряжения в точке электрической сети ниже 0,9 Uном, за которым следует восстановление напряжения до первоначального или близкого к нему уровня за промежуток времени от десяти миллисекунд до нескольких десятков секунд (рис.1).

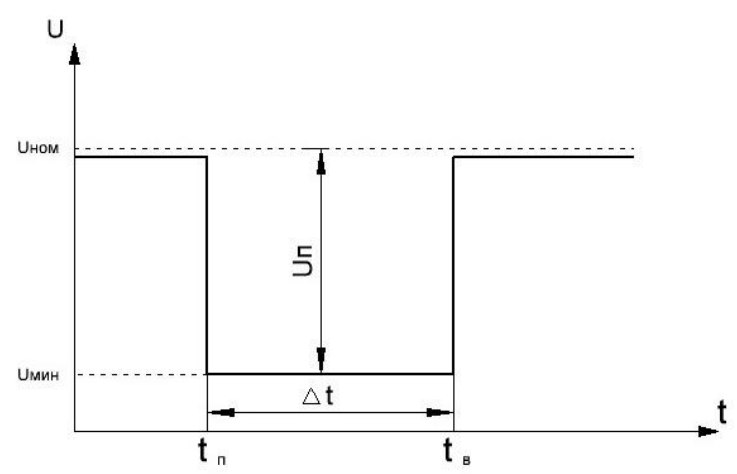

Рисунок 1 - Изображение провала напряжения

6. Импульсное напряжение - резкое изменение напряжения в точке электрической цепи, за которым следует восстановление напряжения до первоначального или близкого к нему уровня за промежуток времени до нескольких миллисекунд (т.е. меньше полупериода); 
7. Временное перенапряжение - увеличение напряжения в точке электрической сети выше 1,1 Uном продолжительностью более 10 мс, возникающее в системах электроснабжения при коммутациях или коротких замыканияX.

Как известно, любые отклонения электрических параметров ухудшают качество электрической энергии, что может нарушить нормальную работу электрического оборудования и даже вывести его строя.

Сложность современного электроэнергетического производства приводят к необходимости измерять и контролировать единовременно сотни физических величин. Главную роль в этом процессе играет устройство первичного сбора и обработки данных.

Основными функциями данного устройства являются: получение измерительной информации от объекта исследования, обработка информации, представление информации оператору или ЭВМ, формирование управляющих воздействий [2]. Рассмотрим обобщенную структуру измерительной информационной системы (ИИС) (рис.2):

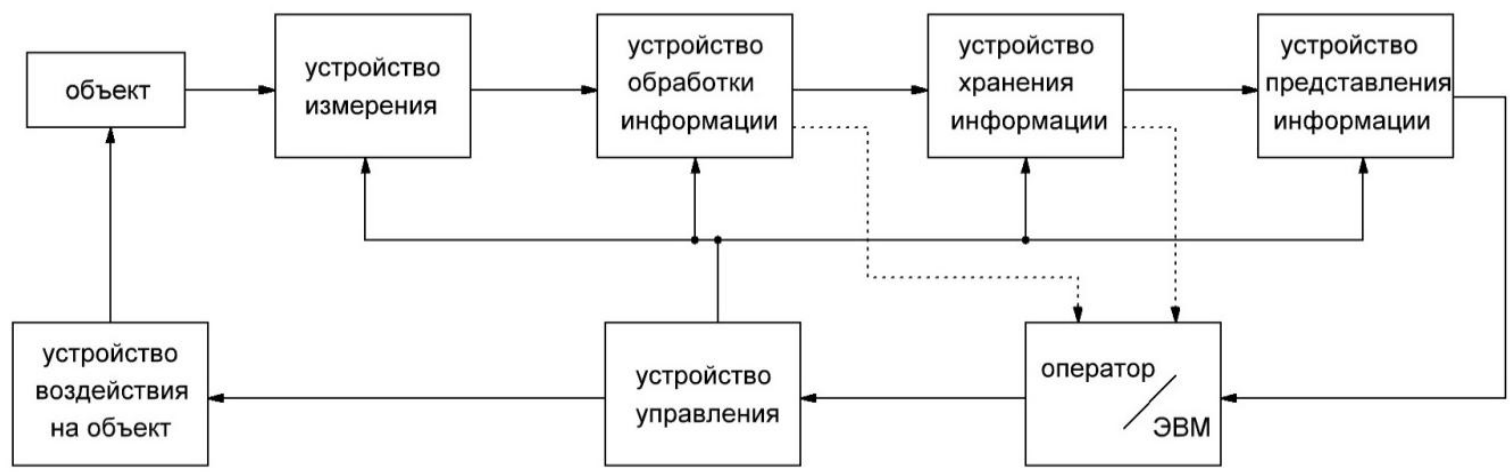

Рисунок 2 - Структура ИИС

Структура ИИС содержит:

- Устройство измерения включает в себя первичные и вторичные измерительные преобразователи и выполняет операции сравнения с мерой, квантование, кодирование, а также может содержать коммутатор;

- Устройство обработки информации выполняет обработку измерительной информации по определенному алгоритму;

- Устройство хранения информации;

- Устройство представления информации - регистраторы, индикаторы;

- Устройство управления, которое служит для организации взаимодействия всех узлов измерительной информационной системы;

- Устройство воздействия на объект, включающее в себя генератор стимулирующих воздействий.

Информация от ИИС выдается оператору или поступает на ЭВМ, которые могут воздействовать на устройство управления ИИС, меняя программу ее работы, и, тем самым, воздействовать на объект.

При разработке средств измерений им присваивают классы точности с учетом результатов государственных приемочных испытаний. Средствам из- 
мерений, предназначенным для измерения двух и более физических величин, присваиваются различные классы точности для каждой измеряемой величины. Единые правила установления пределов допускаемых погрешностей показаний по классам точности средств измерений регламентирует ГОСТ 8.40180 [3]. Классы точности цифровых измерительных приборов со встроенными вычислительными устройствами для дополнительной обработки результатов измерений устанавливают без учета режима обработки.

Для этого определяют два пути повышения точности и достоверности измерений количества электроэнергии [4]:

1. Технологический, основанный на правильном и технически обоснованном выборе приборов учета (замена индукционных счетчиков на электронные, выбор трансформаторов тока (ТT) и напряжения (ТН) в соответствии с требованиями нормативных документов, периодическая проверка ТТ и ТН);

2. Структурный, основанный на методах автоматической компенсации погрешностей и введении поправок при расчетах с учетом индивидуально определенных систематических погрешностей для каждого узла учета.

Комплексное использование данных методов позволяет повысить достоверность и точность измерений и, как следствие, снизить потери электроэнергии. Повышение точности измерительных устройств возможно также за счет повышения точности первичных измерительных данных, на основе которых вычисляются показатели качества электроэнергии. Для достижения поставленных целей предлагается повышение точности устройств первичной обработки информации. Чем точнее будут данные после первичной обработки, тем эффективнее можно выполнить контроль качественных показателей электрической сети.

Проанализировав ряд публикаций [5-7], в которых внимание уделяется интеллектуальным информационным системам контроля показателей качества электроэнергии, вопросам построения средств измерения с повышенной точностью первичных измерительных данных, можно сказать, что данная область достаточно актуальна на сегодняшний день и имеет смысл быть усовершенствованной.

Как указывалось выше, для любой автоматизированной системы контроля и учета энергоресурсов (АСКУЭ) одним из наиболее ответственных узлов, определяющих качество работы и точность первичных измерений, является блок преобразования аналоговой информации в цифровую - АЦП. В процессе аналого-цифрового преобразования практически всегда присутствуют систематические погрешности смещения нуля и коэффициента передачи, подразделяемые, в зависимости от значения входного сигнала, на три группы: аддитивные, мультипликативные и нелинейные [5].

Наличие подобного рода погрешностей приводит к повышению технических потерь в энергетических системах и, в результате, к недоучету (небалансу) электрической энергии. Для уменьшения небаланса в АСКУЭ предлагается встраивать алгоритм цифровой автоматической коррекции погрешностей 
(АКП) АЦП, позволяющий осуществлять коррекцию всех видов погрешностей с заданной точностью [6].

Упрощенная схема реализации метода эталонных сигналов для коррекции погрешности АЦП состоит из измерительного коммутатора (К), подключающего измеряемую величину (х) и эталонные величины (Uэ1,Uэ2), аналого-цифрового преобразователя (АЦП) и вычислителя (В) (рис.3).

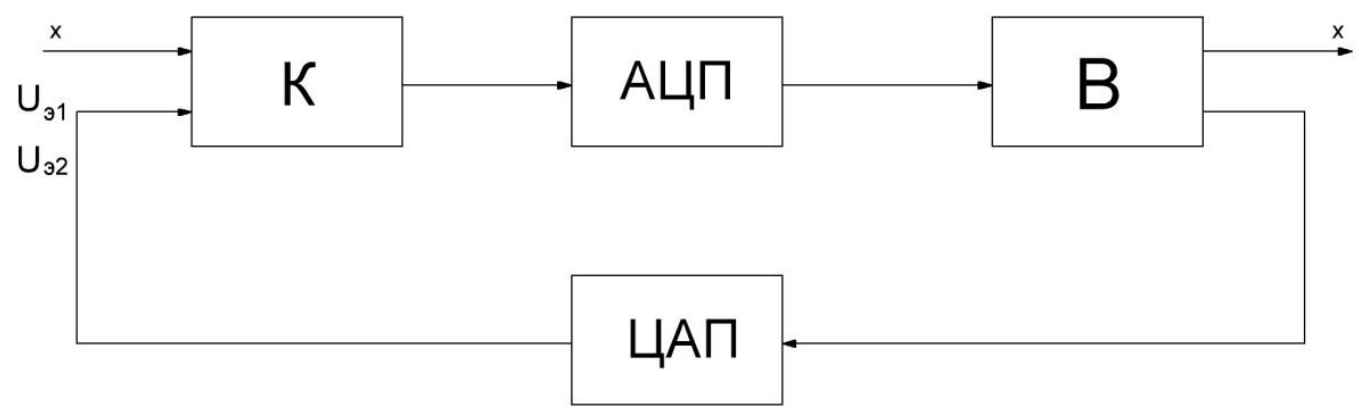

Рисунок 3 - Упрощенная схема реализации алгоритма АКП АЦП

Алгоритм АКП строится на основе аддитивно-мультипликативного алгоритма [7] в сочетании с методом касательных (Ньютона) [8]. Итерационный алгоритм АКП АЦП применим для автоматической коррекции любых видов погрешностей измерительных комплексов. Одновременно с коррекцией погрешности решается задача автоматической идентификации характеристики преобразования измерительного канала в целом.

Рассмотрим способ увеличения точности измерений для интеллектуальных систем учета электроэнергии, основанный на базе данного алгоритма коррекции погрешностей. Повышение точности измерений предполагает использование самых современных технологических средств сбора и обработки информации. Раскроем следующий алгоритм (рис.4):

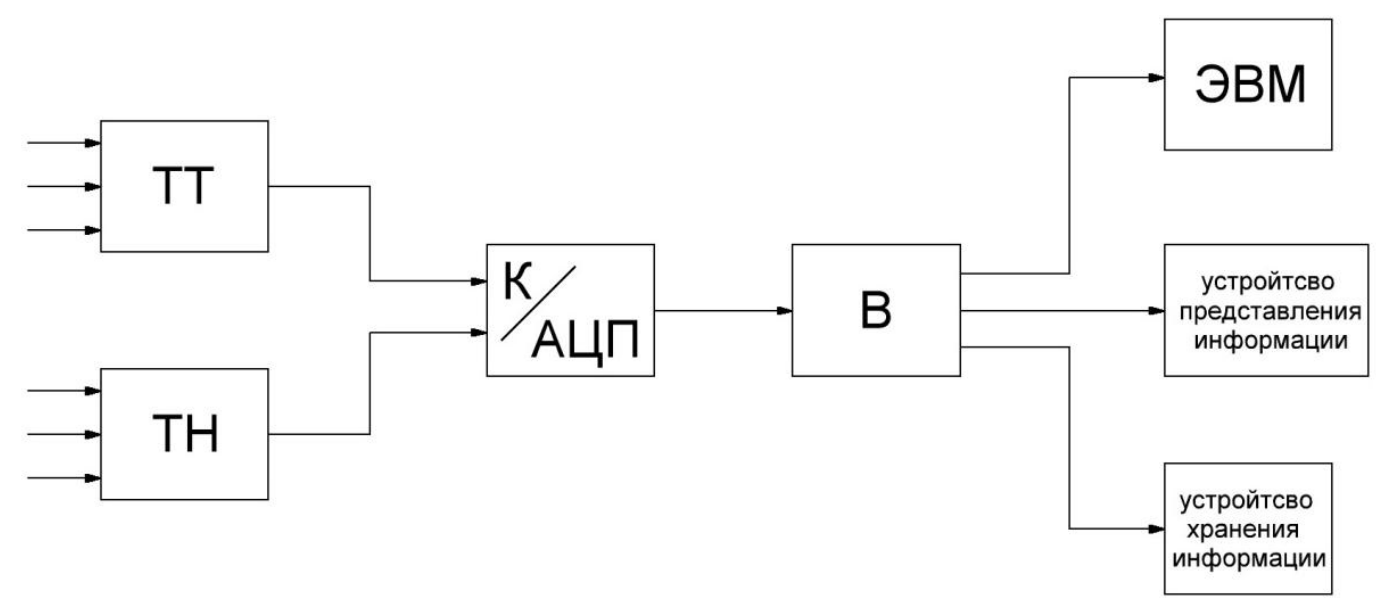

Рисунок 4 - Структурная схема повышения точности сбора первичных данных АСКУЭ

Измеряемые ток и напряжение воздействуют на первичную обмотку измерительного трансформатора тока или напряжения, а вторичная обмотка - 
понижающая, включена на измерительный прибор. В качестве измерительного устройства, с целью повышения точности измерений, был выбран конфигурируемый коммутатор компании Silego Technology SLG6M6001V, позволяющий с высокой точностью измерять протекающий через него ток. Основные достоинства данного прибора, за исключением высокой точности, определяются наличием набора программируемых функций и способностью измерения мгновенной потребляемой мощности [9]. SLG6M имеет ультранизкое сопротивление открытого канала $(3,8$ мОм) и пропускает через себя ток 10 А. Коммутатор является более специализированным устройством в области измерения тока, так как позволяет получить более точную информацию за меньшее время. Как известно, существующие аналого-цифровые преобразователи наделены только одним из двух показателей - либо это быстродействие, либо высокая точность. SLG6M позволяется объединить оба этих качества в одном устройстве, что повысит точность измерений без потери времени.

Оцифрованная коммутатором информация передается на вычислительное устройство, которое занимается выполнением необходимых преобразований данных, сравнивает полученные данные с эталонными значениями показателей качества электроэнергии, обеспечивает управление функциями коммутатора. Вся полученная обрабатываемая и эталонная информация содержится в устройстве хранения информации (встроенная память вычислительного устройства).

Посредством ЭВМ осуществляется управление параметрами коммутатора, а также программирование и алгоритмизация устройства вычисления, в качестве которого предлагается использование сигнальных процессоров компании Analog Devices, отличающихся довольно высокой производительностью. В частности, предлагается использование сигнального процессора ADSP-BF561 Blackfin [10], располагающего достаточно высокой частотой обработки данных - 600 МГц, большой памятью на кристалле - 328 Кбайт, расположенных в виде отдельных систем памяти для каждого ядра, а также высокую пропускную способность. Результаты обработки информации и сравнения с эталонными значениями выводятся на устройство представления информации для мониторинга процесса учета электроэнергии.

Вывод. Применение методов технологического и структурного повышения точности на базе программно-алгоритмических средств автоматической коррекции погрешностей позволит повысить интеллектуальный уровень автоматизированных систем контроля и учета электроэнергии, качество и точность измерений, а также достоверность учета электрической энергии в энергетических системах.

\section{Библиографический список:}

1. ГОСТ 13109-97. Электрическая энергия. Совместимость электрических средств электромагнитная. Нормы качества электрической энергии в системах электроснабжения общего назначения 
2. Банкин С.А. Измерительные информационные системы в электроэнергетике. / Банкин С.А., Щуревич В.А. // Алтайский государственный технический университет. Ползуновский альманах. -2004. -№1. -С.18-28.

3. ГОСТ 8.401-80. Государственная система обеспечения единства измерений. Классы точности средств измерений. Общие требования.

4. Загорский Я.Т. Совершенствование метрологического обеспечения измерений и учета электроэнергии и мощности на межсистемных перетоках по межгосударственным ЛЭП стран СНГ [Электронный ресурс] // Итоги 23-го заседания Электроэнергетического Совета. - г. Чолпон-Ата -2003. URL www.fsk-ees.ru/common/img/uploaded/sp-3-67.doc

5. Набиева Е.Б. Повышение точности контроля учета электрической энергии в энергетических системах / Набиева Е.Б. // Вести высших учебных заведений черноземья. - 2008. -№2(12). -С.3-5.

6. Андрианова Л.П. Цифровая автоматическая коррекция погрешностей микропроцессорных систем учета электроэнергии / Андрианова Л.П., Набиева Е.Б. // Измерительная техника. 2003. - № 7. - С.7-10.

7. Прохоренко А.М. Методы и средства повышения качества функционирования информационно-управляющих комплексов / И.В. Сабуров, Е.И. Сабуров // Современные наукоемкие технологии. - 2005. -№ 10. - С.88-89.

8. Чивилихин С.А. Вычислительные методы в технологиях программирования. Учебное пособие. С-П: Редакционно-издательский отдел СанктПетербургского государственного университета информационных технологий, механики и оптики, 2008. -110с.

9. Техническая документация силового ключа SLG6M6001V. [Электронный pecypc] // URL

www.silego.com/uploads/Products/product_331/SLG6M6001_DS_r070_04212015 .pdf

10. Техническая документация цифрового сигнального процессора ADSPBF561. [Электронный ресурс] // URL

www.analog.com/media/en/technical-documentation/data-sheets/ADSP-BF561.pdf 